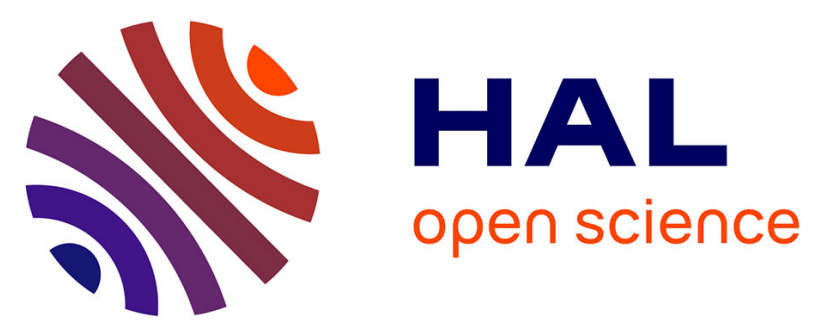

\title{
3D Sequential Kinematics of the Femoro-Tibial Joint of Normal Knee from Multiple Bi-planar X-rays: Accuracy and Repeatability
}

\author{
Karine Langlois, Hélène Pillet, François Lavaste, Goulven Rochcongar, \\ Philippe Rouch, Patricia Thoreux, Wafa Skalli
}

\section{To cite this version:}

Karine Langlois, Hélène Pillet, François Lavaste, Goulven Rochcongar, Philippe Rouch, et al.. 3D Sequential Kinematics of the Femoro-Tibial Joint of Normal Knee from Multiple Bi-planar X-rays: Accuracy and Repeatability. Innovation and Research in BioMedical engineering, 2018, 39 (4), pp.251260. 10.1016/j.irbm.2018.05.001 . hal-02483403

\section{HAL Id: hal-02483403 https://hal.science/hal-02483403}

Submitted on 18 Feb 2020

HAL is a multi-disciplinary open access archive for the deposit and dissemination of scientific research documents, whether they are published or not. The documents may come from teaching and research institutions in France or abroad, or from public or private research centers.
L'archive ouverte pluridisciplinaire HAL, est destinée au dépôt et à la diffusion de documents scientifiques de niveau recherche, publiés ou non, émanant des établissements d'enseignement et de recherche français ou étrangers, des laboratoires publics ou privés. 


\title{
3D Sequential Kinematics of the Femoro-Tibial Joint of Normal Knee from Multiple Bi-planar X-rays: Accuracy and Repeatability
}

\author{
K. Langlois ${ }^{\text {a,* }}$, H. Pillet ${ }^{\mathrm{a}, *}$, F. Lavaste ${ }^{\mathrm{a}}$, G. Rochcongar ${ }^{\mathrm{b}}$, P. Rouch ${ }^{\mathrm{a}}$, P. Thoreux ${ }^{\mathrm{a}, \mathrm{c}}$, W. Skalli ${ }^{\mathrm{a}}$ \\ a Arts et Métiers ParisTech, LBM/Institut de Biomécanique Humaine Georges Charpak, 151 bd de l'Hôpital, 75013 Paris, France \\ b INSERM U1075 COMETE “Mobilité: Attention, Orientation \& Chronobiologie”, Département de Chirurgie Orthopédique et Traumatologique, \\ Université de Caen Basse-Normandie, Centre Hospitalier Universitaire de Caen, France \\ ${ }^{\mathrm{c}}$ Service de Chirurgie Orthopédique Hôpital Avicenne, Université Paris XIII, 125 rue de Stalingrad, 93009 Bobigny, France
}

\section{Highlights}

- The EOS system allows the sequential kinematic analysis of the femoro-tibial joint.

- The reliability of the registration of $3 \mathrm{D}$ model on $2 \mathrm{D}$ views has been quantified.

- The accuracy of the registration is inferior to $1.6 \mathrm{~mm}$ and $0.4^{\circ}$.

- The repeatability of the registration is $0.3^{\circ}, 2.1^{\circ}$ and $1.6^{\circ}$, for the rotations.

- The repeatability of the registration is inferior to $1.8 \mathrm{~mm}$ for the translations.

\section{Graphical abstract}

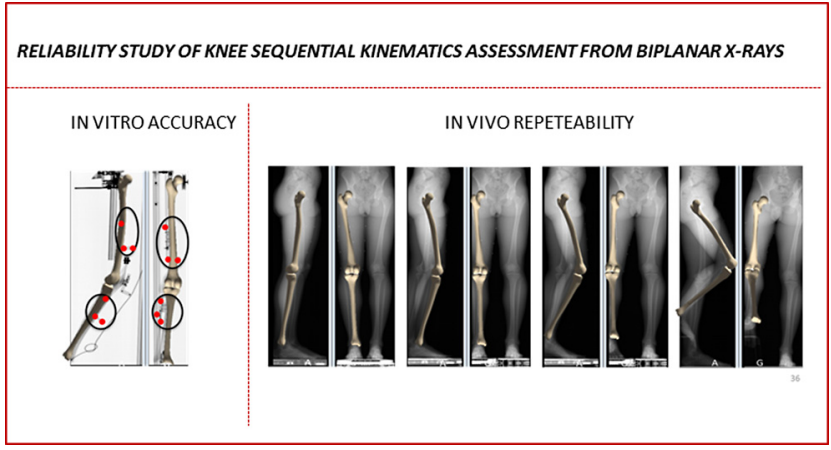

\section{Abstract}

Background: Several methods can be used to assess joint kinematics going from optoelectronic motion analysis to biplanar fluoroscopy. The aim of the present work was to evaluate the reliability of the use of biplane radiography to quantify the sequential 3D kinematics of the femoro-tibial joint.

Methods: Bi-planar X-rays (EOS imaging) of 12 lower limbs (6 specimens in vitro and 6 subjects in vivo) were taken for various knee flexion angles. 3D personalized models of the femur and the tibia were registered on each pair of views. To quantify the bias, the kinematic parameters calculated from the registered models were compared to those obtained from the tripods embedded in the specimens. Intra and inter-operator repeatability of each parameter were assessed from the registrations made by 3 operators in vivo.

Results: In vitro, the bias of the tibia pose estimation obtained from the registration method was inferior to $1.6 \mathrm{~mm}$ and $0.4^{\circ}$. In vivo, the repeatability of the sequential kinematic parameters was inferior to $0.3^{\circ}, 2.1^{\circ}$ and $1.8^{\circ}$, for respectively flexion, varus-valgus and medial-lateral rotation and inferior to $1.8 \mathrm{~mm}$ for translations. 
Conclusion: Compared to simple fluoroscopy, the accuracy of our method based on sequential images was of the same order of magnitude, with better results for the translation in the frontal plane. The low dose of radiation of the EOS system offers promising prospects for a clinical use of this method to assess the femoro-tibial sequential kinematics.

Keywords: Bi-planar radiography, low dose; Knee 3D sequential kinematics; Registration; Lower limb

\section{Introduction}

The assessment of 3D femoro-tibial joint kinematics is essential to understand the complex function of the knee and the mechanism of degeneration. Indeed, some studies have already shown that joint disorders such as osteoarthritis affect these kinematics [1,2]. However, in vivo extensive evaluations remain difficult due either to the radiant or invasive nature of direct bone localization techniques [3-8] or to inaccuracies associated to external measurement procedures like optical motion capture [9-11]. Thus, the registration on 2D fluoroscopic images of a 3D model obtained from CT-scan is so far considered as the gold standard for the capture of skeletal motion and the accuracy of the method has been evaluated through in vitro studies $[4,12,13]$. Considering that this technique exposes the subject to a high level of radiation, an alternative has been proposed taking advantage of magnetic resonance imaging (MRI) to visualize bones during sequential knee joint motion. To overcome the drawback of the lying position, some loading apparatus have been developed [14-16] and a protocol combining supine and upright MRI data [17] has recently been proposed. However, the technic suffers from several drawbacks: the acquisition time is long, the confine environment makes it difficult to reproduce realistic weight-bearing on the lower limbs, the restricted field of view limits the available information and the image processing remains time consuming [18].

Currently, 3D modeling from low dose bi-planar radiographs (BPR) is able to produce a 3D subject specific reconstruction of the lower limbs from calibrated images in weight-bearing condition $[19,20]$. The shape reconstruction algorithm relies on the identification of several morphological features on the radiographs and has been validated against CT-scan-based model for both shape and derived morphological parameters [19,21]. Several clinical applications have been investigated such as cerebral palsy [22], knee osteoarthritis [23] and hip osteoarthritis [24]. Nevertheless, these analyses were performed in a standing posture, e.g. with an extended knee, and did not provide any information about the joint kinematics.

In the same time, sequential analysis of the femoro-tibial joint motion was proposed using multiple stereoradiographic images of the considered joint in different knee flexion poses. This kind of analysis requires the registration of the 3D personalized model on each pair of images acquired by the BPR corresponding to one specific flexion pose of the joint. The registration can be made manually and femoro-tibial joint kinematics derived from this procedure were previously used as a reference to evaluate and compare several tracking methods with marker cluster [25] or to test an automatic process based on image processing [26]. Some authors also proposed a semiautomatic method based on image processing to perform this registration, which has been evaluated in two studies [27,28]. However, in these studies, the quantification of the accuracy of the method relied on the use of simulated radiographs of 3D models virtually positioned in different configurations. On the contrary, some authors assessed the accuracy of similar registration technique through in vitro study for the patellofemoral joint [29] and the scapulo-humeral joint [30]. It remains to be quantified in the context of the femoro-tibial joint motion. Therefore, there is a need to perform a complete validation study to assess the accuracy and the repeatability of the 3D femoro-tibial motion quantification from sequential bi-planar images of the joint. Indeed, the reliability of the sequential kinematic parameters directly depends on the reliability of the registration method. The hypothesis of the current study was that biplane radiography can be used to reliably assess femoro-tibial sequential kinematics in weight bearing conditions.

In this framework, the aim of the study was to estimate the uncertainties of the 3D sequential analysis of the kinematics of the femoro-tibial joint obtained from bi-planar radiographies by quantifying the registration reliability.

\section{Methods}

To quantify the reliability of the registration of 3D models on $2 \mathrm{D}$ views, two sets of data collected in vitro and in vivo were used. First, the accuracy of the registration was assessed using an in vitro data set to compare the registration to reference values derived from marker clusters screwed in the bones. Second, the intra and inter-observer repeatabilities of the registration method were quantified from an in vivo data set. Both accuracy and repeatability of sequential kinematic parameters of the femoro-tibial joint were calculated.

\subsection{In vitro analysis}

Six human cadaveric lower limb specimens were harvested after approval of the ethical committee of the laboratory of Anatomy of Caen University (Normandie, France).

Each specimen included the femur, the patella, the fibula, the tibia and the intact joint passive structures. The ages of donors were between 47 and 79 years old. Specimens were checked regarding trauma, surgery and evident deformity and were fresh frozen, then thawed at room temperature during a twenty-four hour period. Clusters made of three retro-reflective markers each were screwed in the femur, the tibia and the patella in order to get reference values for motion tracking. The specimens were 


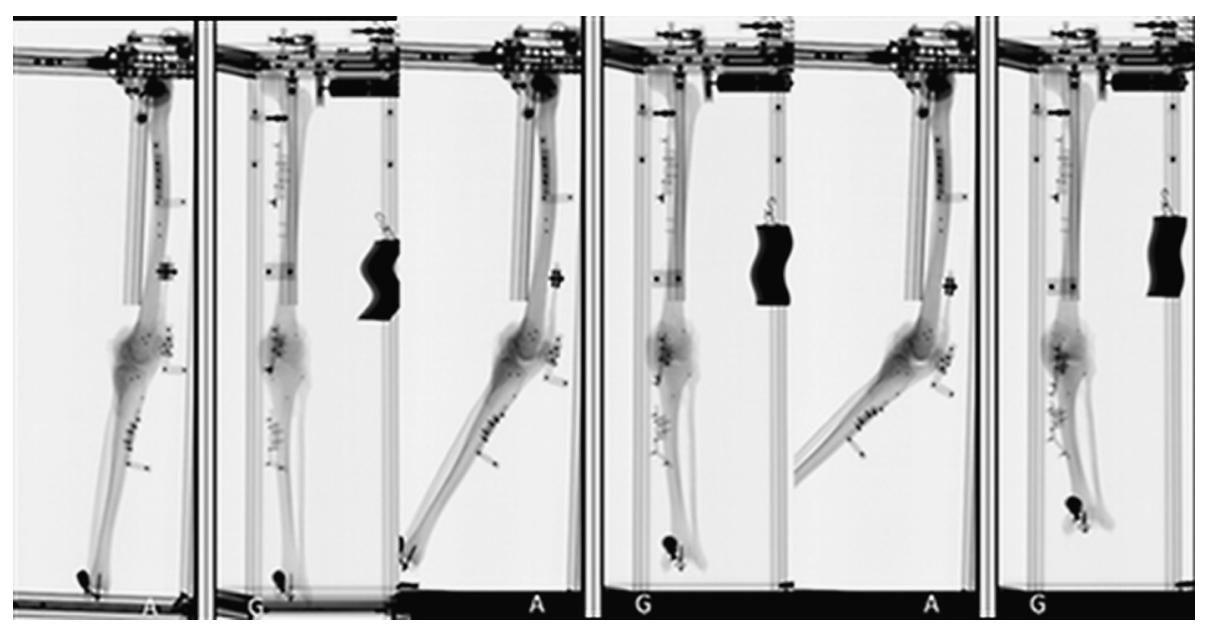

Fig. 1. In vitro views in extension pose, in $20^{\circ}$ and $40^{\circ}$ flexion poses. Cluster markers in each bone are also identifiable.

set in a device adapted from Azmy et al. [31] and described previously [32] (Fig. 1). In this device, the femur was fixed and the tibia was free to move. Knee flexion was induced by pulling the extremity of the tibial pylon in order to reach successively $20^{\circ}$ and $40^{\circ}$. Beyond $40^{\circ}$ of flexion, as the femur was fixed in the device, the inferior part of the tibia was not visible anymore in the measurement space. As the registering method consists in matching skeleton contours of the 3D model with visible ones on the images, it could therefore not be evaluated for flexion angles higher than $40^{\circ}$. For the three knee poses (extended and two flexion angles) two orthogonal digital radiographies were simultaneously acquired using low dose bi-planar radiographs $\left(\right.$ EOS $^{\circledR}$, EOS-imaging, France).

\subsection{In vivo analysis}

Six asymptomatic volunteers without any history of knee trauma participated in the study after approval by the relevant Ethics Committee (CPP 06036, Hospital Pitie Salpetriere Paris). The characteristics of the volunteers were in average 35.7 years old $(\mathrm{SD}=10.3$ years old $), 173 \mathrm{~cm}(\mathrm{SD}=10 \mathrm{~cm})$ and $67 \mathrm{~kg}(\mathrm{SD}=9.5 \mathrm{~kg})$. The femoro-tibial angle, in the coronal plane, of their left lower limb was in average $175.4^{\circ}(\mathrm{SD}=$ $1.4^{\circ}$ ), which corresponds to typical values of healthy subjects. Firstly, an acquisition with the same bi-planar radiographic system $\left(\mathrm{EOS}^{\circledR}\right.$, EOS-imaging, France) was taken in standing condition according to the procedure described by Chaibi [19]. Each volunteer was then asked to stand in the EOS cabin taking successively three different angular poses of the left knee corresponding to approximately $20^{\circ}, 40^{\circ}$ and $90^{\circ}$ of flexion (Fig. 2). For the $20^{\circ}$ knee flexion pose, the foot remained flat. For the $40^{\circ}$ knee flexion pose, the heel was off so that the toes were resting on the ground. For these last two knee flexion poses, the partial loading on the flexed lower limb was quantified by positioning a Wii balance board (WBB, Nintendo ${ }^{\circledR}$, Japan) under the foot. For the $90^{\circ}$ knee flexion pose, the forefoot was supported on a step. Femur and tibia were mobile between the different knee flexion angles. Compared to the in vitro protocol, the in vivo analysis included an additional pose ( $90^{\circ}$ flexion). This is due to the clinical relevance of this con- dition in vivo for the study of knee pathologies which ought to modify the kinematics during high amplitude motions. Flexion angles were manually controlled with a goniometer. For each view, the radiation dose could be calculated by the value of the emitted dose and the surface of the body that was irradiated. Thus, the average dose for the 4 positions (FSP and 3 flexion positions) corresponding to 8 stereoradiographies could be quantified.

\subsection{Digital bones models: reconstruction and registration}

From both in vitro and in vivo acquisitions, for each considered knee, 3D subject specific models of the femur and the tibia were obtained by using a reconstruction algorithm described and validated in previous works from the pair of views of the extended knee pose [19,33]. These 3D subject specific models then served in a procedure of manual 2D-3D rigid registration of the femur and the tibia on each pair of views for each knee flexion pose $\left(20^{\circ} ; 40^{\circ}\right.$ and $\left.90^{\circ}\right)$ (the views and steps of the method in the "in vivo conditions" appear on Figs. 2 and 3). Both tibial and femoral models were projected into the $2 \mathrm{D}$ flexion views and their positions progressively adjusted so that the contours of the projected models match the bones edges visible on the X-rays images. Three fully trained operators (one surgeon, one physiotherapist and one engineer) contributed to this study performing the registrations of the in vitro specimens and two of them (the surgeon and the physiotherapist) also performed the in vivo registrations. For each knee (in vitro and in vivo), the operators performed three repetitions of the overall process of reconstruction and registration.

In addition, for in vitro X-rays, the markers of each cluster were identified on bi-planar X-rays and the $3 \mathrm{D}$ coordinates of the centroid of each marker were obtained for each flexion pose, thus providing reference data for the accuracy analysis.

\subsection{Assessment of the sequential kinematics of the femur and the tibia}

From 3D personalized models of the femur and the tibia, anatomical frames were computed and attached to the bones 


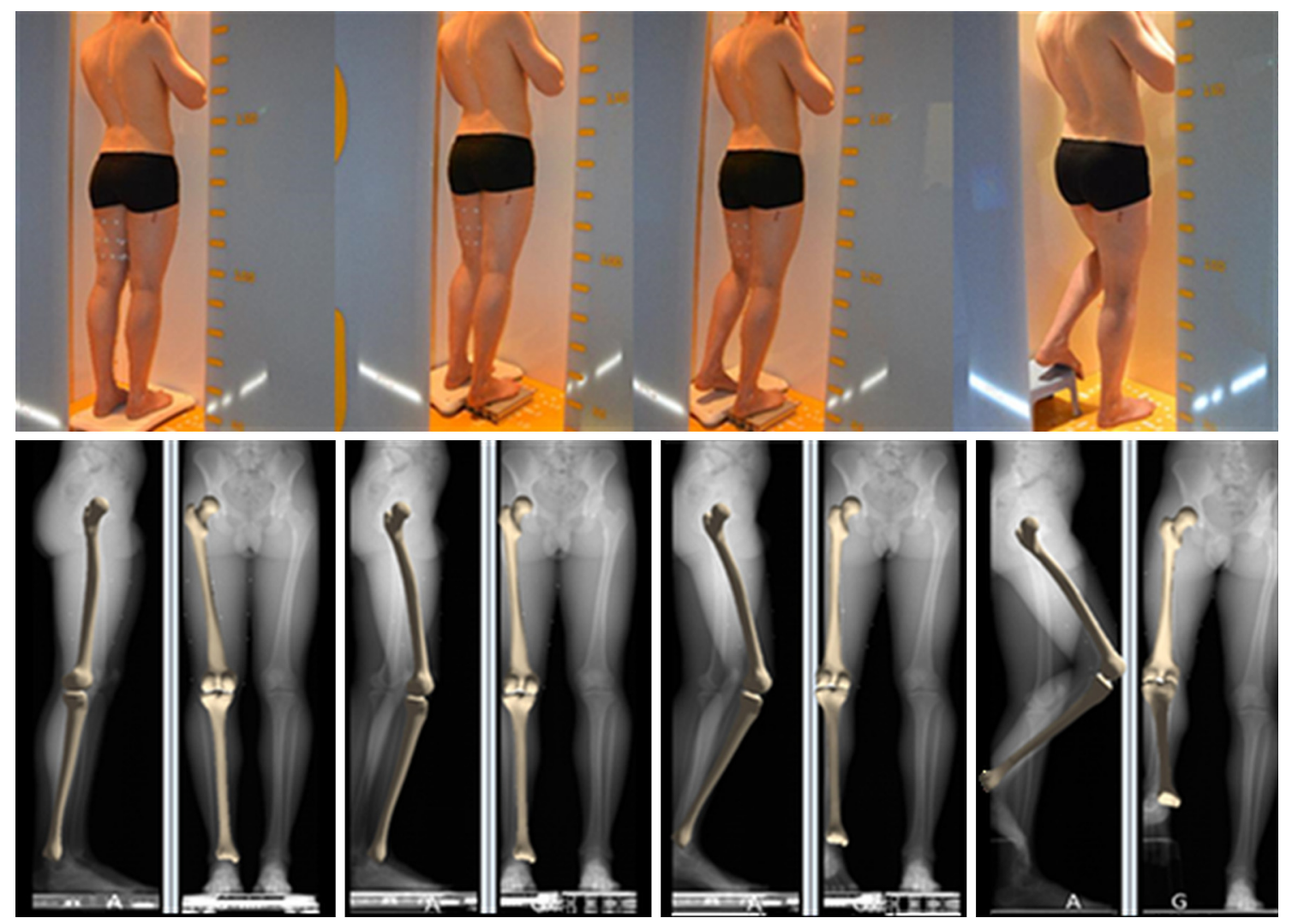

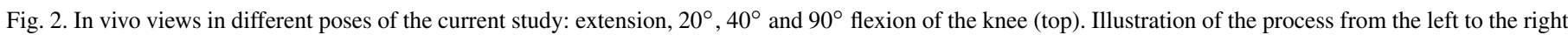

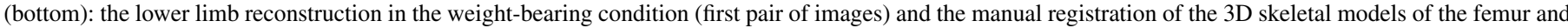
the tibia on each position of the knee flexion.
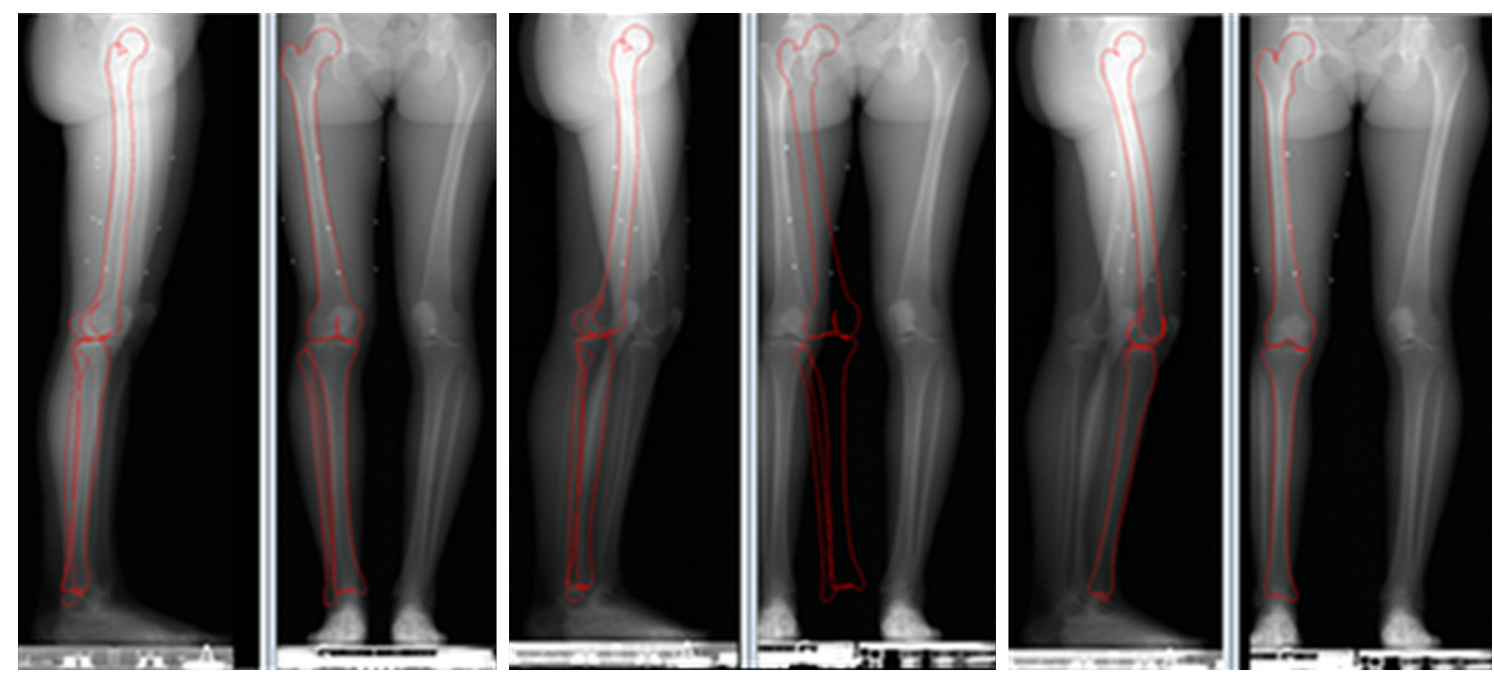

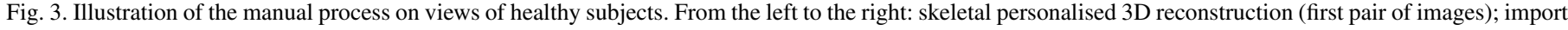

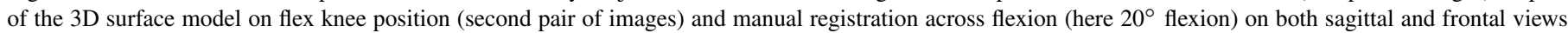
(third pair of images).

according to the definition proposed by Schlatterer [34] for each knee position and each repetition. First, the variations of position and orientation of each bone relatively to the EOS coordinate system was assessed for each knee flexion pose relatively to the extended knee pose. They were expressed by 6 parameters per bone: anteroposterior (x), longitudinal (y) and mediolateral (z) translations (Tx, Ty, Tz) and rotations ( $\mathrm{Rx}, \mathrm{Ry}$, $\mathrm{Rz})$. Secondly, the variation of the position and orientation of the tibia relatively to the femur was also calculated between the different knee flexion poses giving also 6 parameters per pose. These parameters characterize the angular and linear sequential kinematics of the femoro-tibial joint. 


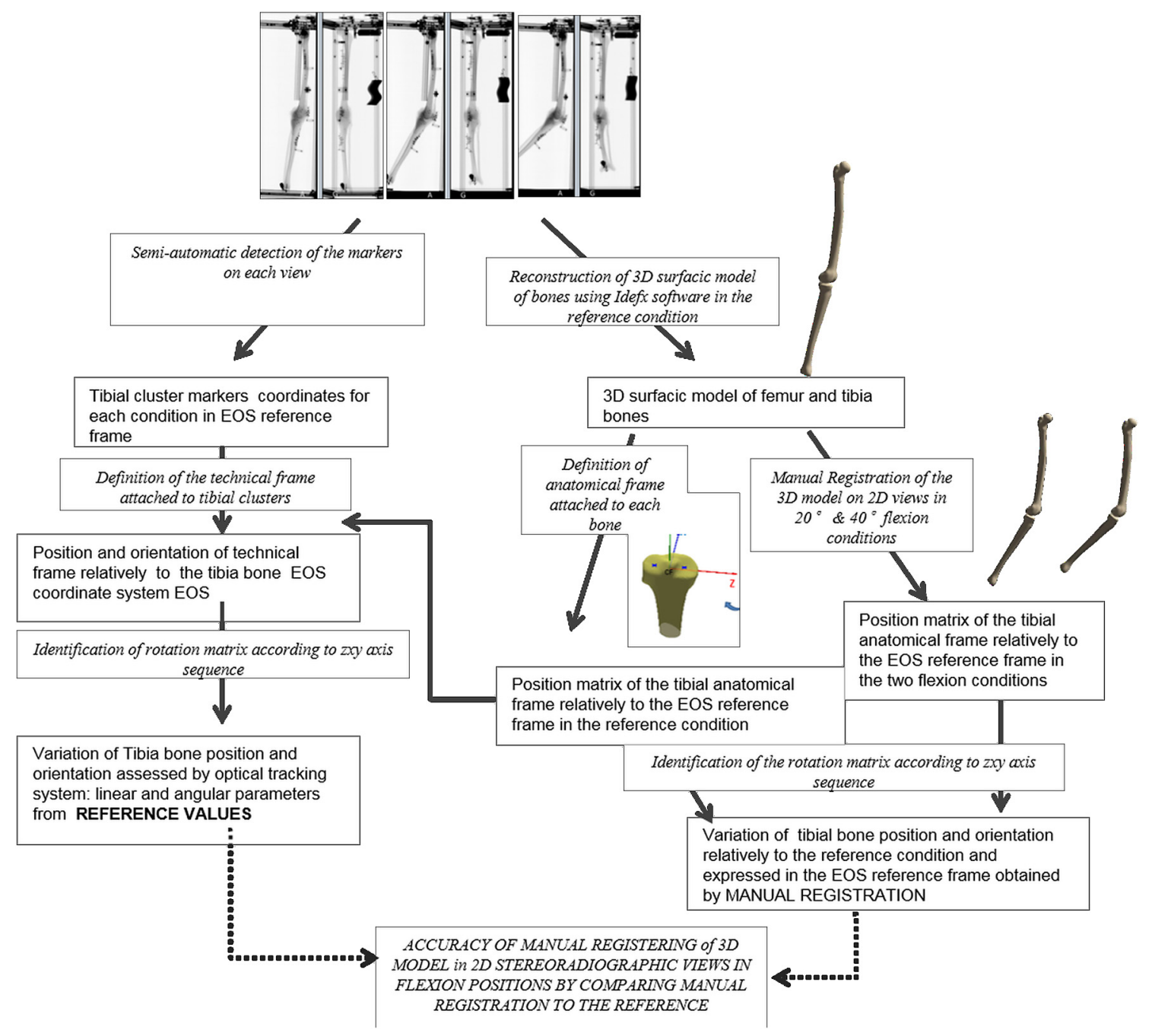

Fig. 4. Graph illustrating the method to compare both kinematics assessment techniques.

The three rotation angles were obtained using the Cardan convention and the sequence "ZXY" from equivalence between both position and rotational matrices. The linear parameters were obtained by computing the variation of the position of the center of the femur frame expressed in the tibia frame, between two successive poses. The angular parameters characterized the relative position of the tibia frame expressed in the femur frame.

In summary, eighteen parameters were calculated to characterize the sequential motion between the extended knee pose and each flexion pose $\left(20^{\circ}, 40^{\circ}, 90^{\circ}\right.$ in vivo and $20^{\circ}$ and $40^{\circ}$ in vitro).

In the same time, the same variations of position were also assessed from the clusters embedded in each bone. These clusters were used to define technical frames. Then, the mathematical relation between anatomical and technical frames was obtained and used as an invariant to calculate the position of the bone from the position of the cluster. The parameters obtained through this procedure were considered as a reference since the identification of markers has been proven to be highly repeatable [35].

\subsection{Estimation of the reliability of the method}

The reliability of the registration method was characterized from both the accuracy and the repeatability of the sequential kinematic parameters obtained from this method.

\subsubsection{Assessment of the accuracy of the tibia registration from in vitro data}

For each knee ( 6 specimens) and each flexion pose $\left(20^{\circ}\right.$ and $40^{\circ}$ ), the accuracy (bias) of the tibia registration was evaluated by comparison of reference data (from clusters of markers) to data obtained using the registration procedure.

Thus, for each parameter, the bias was calculated as the difference between the values of the reference data derived from clusters and those calculated from the registration technique after averaging the results of the different operators and repetitions (Fig. 4).

\subsubsection{Assessment of the repeatability of the registration}

From femur and tibia kinematic parameters, the reliability of the registration method was assessed according to the ISO 
Table 1

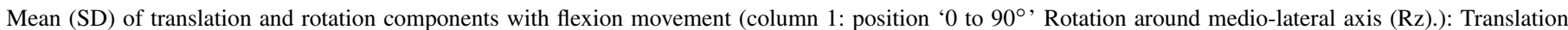
along posterio-anterior axis (Tx), disto-proximal axis (Ty), and medio-lateral axis (Tz). Rotation around postero-anterior axis (Rx) and disto-proximal axis (Ry).

\begin{tabular}{|c|c|c|c|c|c|}
\hline & Tx mm & Ty mm & $\mathrm{Tz} \mathrm{mm}$ & $\mathrm{Rx}^{\circ}$ & $\mathrm{Ry}^{\circ}$ \\
\hline \multicolumn{6}{|l|}{ Knee flexion angle: $\mathrm{Rz}^{\circ}(\mathrm{SD})$} \\
\hline Position ' $0^{\circ}$ ' $3.8^{\circ}(3)$ & $-6.4(1.6)$ & $23.8(1.7)$ & $3.9(1.5)$ & $-0.4(1.9)$ & $7.2(3.3)$ \\
\hline Position ' $40^{\circ}:-42.5^{\circ}(4.2)$ & $-6.7(2.7)$ & $21.7(2.1)$ & $4.2(1.7)$ & $-3.4(2.9)$ & $-11.9(3.9)$ \\
\hline Position ' $90^{\circ}:=80.1^{\circ}(3.2)$ & $-9.6(1.7)$ & $21(3)$ & $4.4(1.6)$ & $-3.7(2.9)$ & $-11.1(3.2)$ \\
\hline
\end{tabular}
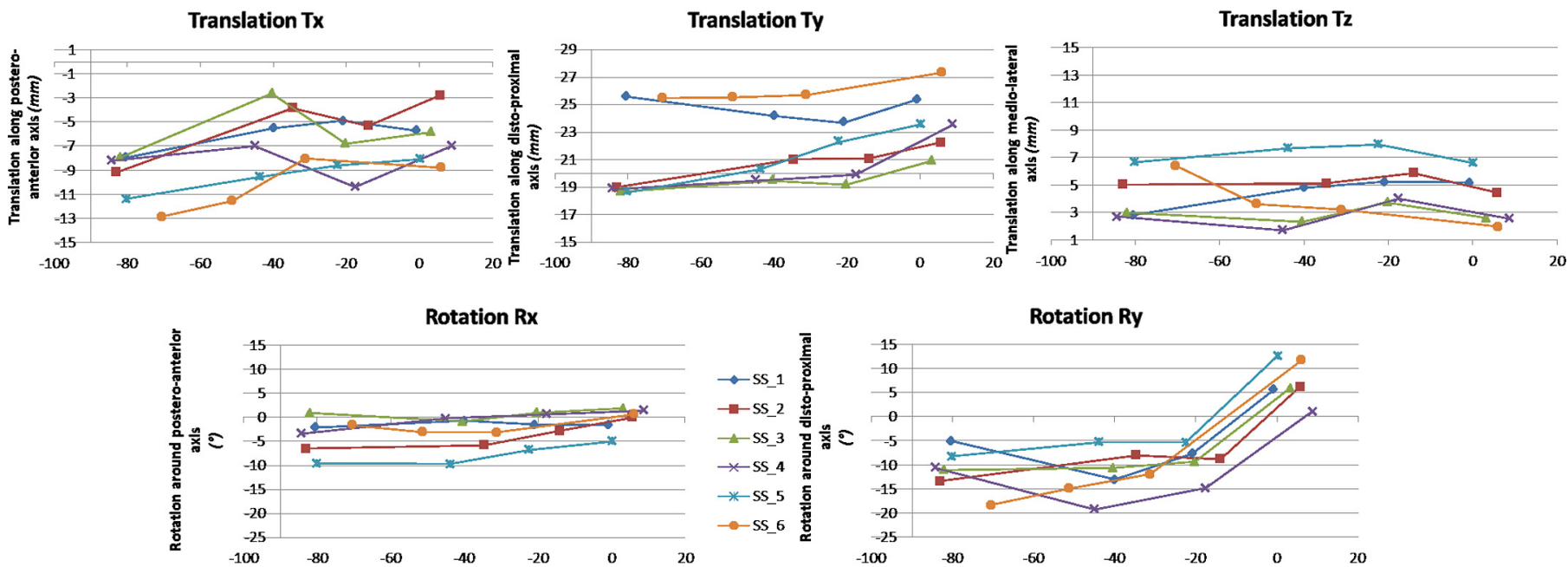

Fig. 5. Translations parameters (top of the figure) of the center of the femur frame along postero-anterior $x$ axis, disto-proximal $y$ axis, medio-lateral $z$ axis and according to the three knee positions (pos_2 $20^{\circ}$; pos_ $40^{\circ}$; pos_ $90^{\circ}$ ). Rotation parameters (bottom of the figure) of the femur relatively to tibia around postero-anterior $x$ axis (positive values for knee abduction/negative values for knee adduction) and around disto-proximal $y$ axis (positive values for knee medial rotation/negative values for knee lateral rotation) and according to the three knee flexion angle (pos_20 ; pos_40 ; pos_ $90^{\circ}$ ).

5725-2 standard (International Organization for Standardization (ISO), 1994) and involved both intra- and inter-observers repeatability variances.

The ISO recommends to estimate the intra observer repeatability as the average over the operators of the variances from all repetitions $\left(S_{r i}^{2}\right)$ for each kinematic parameter of one subject $(i)$.

The inter-observer variance represents the variance of mean values obtained by all operators $\left(S_{L i}^{2}\right)$. Then, the global variance $\left(S_{R i}^{2}\right)$ was calculated:

$S_{R i}^{2}=S_{r i}^{2}+S_{L i}^{2}$

Finally, a global repeatability estimator (SR) considering all the subjects was calculated as follows:

$S_{R}=\frac{\sqrt{\sum_{i=1}^{n} S_{R i^{2}}}}{n}$

where $i$ is the $i$ th subject and $n$ the number of subjects.

\subsection{In vivo sequential kinematics of the femoro-tibial joint}

Thanks to both angular (position of the tibia relative to the femur), and linear (position of the center of the femur frame expressed in the tibia frame) parameters, the sequential kinematics were given for each knee pose.

\section{Results}

\subsection{In vivo sequential kinematics of the femoro-tibial joint}

Quantified by Wii ${ }^{\circledR}$ platform in the EOS system, the loading on the flexed left lower limb was respectively, 44\% (SD: 5\%) and $31 \%$ (SD: $5 \%$ ) of the body weight in the $20^{\circ}$ and $40^{\circ}$ flexion poses. These values correspond in average to $285 \mathrm{~N}$ (SD: $7 \mathrm{~N}$ ) at $20^{\circ}$ and $213 \mathrm{~N}(\mathrm{SD}: 55 \mathrm{~N})$ at $40^{\circ}$ knee flexion. In average over the 6 subjects, the flexion angles calculated from the 3D models were respectively $-4^{\circ}\left(\mathrm{SD}: 3^{\circ}\right), 21^{\circ}\left(\mathrm{SD}: 4^{\circ}\right), 42^{\circ}\left(\mathrm{SD}: 4^{\circ}\right)$ and $80^{\circ}\left(\mathrm{SD}: 3^{\circ}\right)$ for the targeted flexions of $0^{\circ}, 20^{\circ}, 40^{\circ}$ and $90^{\circ}$.

The average radiation dose produced by the system over all the subjects was $1.18 \mathrm{mGy}$ (SD: $0,28 \mathrm{mGy}$ ) for the entire protocol (4 positions). The average of femoro-tibial joint sequential kinematic parameters over the six subjects is given in Table 1. The evolution of each sequential kinematic parameter according to the flexion angle, for each subject (SS_1 to SS_6) is illustrated on the Fig. 5.

Results show that knee flexion is characterized by an internal rotation (Ry) and by a slight posterior displacement (Tx) simultaneous to the flexion angle $(\mathrm{Rz})$.

\subsection{In vitro accuracy}

The bias estimated from the in vitro study is presented in Table 2. 
Table 2

Bias of the estimation of the reference tibial pose from the manual registration.

\begin{tabular}{lllll}
\hline \multirow{5}{*}{} & & \multicolumn{3}{l}{$\begin{array}{l}\text { In vitro accuracy } \\
\text { Tibia }\end{array}$} \\
\cline { 3 - 5 } & & Global & $20^{\circ}$ & $40^{\circ}$ \\
\hline Linear parameters & $\mathrm{Tx}(\mathrm{mm})$ & 1.2 & 0.9 & 1.5 \\
& $\mathrm{Ty}(\mathrm{mm})$ & 1.6 & 0.8 & 2.4 \\
& $\mathrm{Tz}(\mathrm{mm})$ & -0.3 & 0.0 & -0.5 \\
Angular parameters & $\operatorname{Rx}\left({ }^{\circ}\right)$ & 0.3 & 0.2 & 0.4 \\
& $\operatorname{Ry}\left({ }^{\circ}\right)$ & -0.4 & -0.6 & -0.2 \\
& $\operatorname{Rz}\left(^{\circ}\right)$ & -0.1 & -0.2 & 0.0 \\
\hline
\end{tabular}

\subsection{Repeatability from in vivo data}

The SD repeatability $\left(S_{\mathrm{R}}\right)$ of the registration method was calculated for the assessment of the variations of respectively the tibia absolute position, the femur absolute position and the tibia position relatively to the femur between the different knee poses. Detailed results are presented in Table 3. Results are shown for each knee flexion pose and for each translation and rotation parameter. Regarding angular parameters, the femur and tibia registration highlighted more reliable results around the medio-lateral axis with CI $95 \%$ inferior to $0.6^{\circ}$. About the translation results, the femur repositioning is homogeneous along the three axes with CI $95 \%$ inferior to $1.8 \mathrm{~mm}$. The tibia registration is slightly less reliable than the femur one both in translation (CI 95\% inferior to $2.8 \mathrm{~mm}$ along $\mathrm{z}$ axis) and rotation.

\section{Discussion}

The goal of this study was to evaluate the reliability of a method to analyse the 3D kinematics of the femoro-tibial joint from a sequence of bi-planar radiographic images. The method is based on the manual registration of the $3 \mathrm{D}$ models of the femur and the tibia bones. The accuracy of the method was assessed in vitro and the repeatability was quantified in vivo.

\subsection{Femoro-tibial knee kinematic patterns}

Linear and angular parameters obtained for the 6 knees in vivo represent a reference data base of the sequential kinematics of the femoro-tibial joint of an asymptomatic population. The translations and rotations for the 6 subjects of the study are illustrated as a function of the knee flexion angle in Fig. 5. Indeed, in the literature, few studies gave accurate quantification of femoro-tibial joint kinematics in similar conditions. That is to say: 1/ asymptomatic knees [2,14,36-38], 2/ direct analysis of bone kinematics (opposite to skin marker based kinematics), $3 /$ semi-active flexion under partial loading for the $20^{\circ}$ and $40^{\circ}$ knee flexion poses.

Concerning the axial rotation of the tibia during knee flexion, we found a medial rotation motion for the majority of the subjects between the extended and the $20^{\circ}$ knee flexion poses with a range (average of $16^{\circ}$ ) greater than the method uncertainties. In comparison, previous studies revealed controversial results as concerns the range of the medial rotation. Akbarshahi et al. measured for 3 out of 4 subjects a slight medial rotation which essentially occurred around $20^{\circ}$ of flexion [36]. Farrokhi et al. quantified a medial rotation of $6^{\circ}$ during the stance phase of slope descent when the knee is flexed at $20^{\circ}$ [2]. Benoit et al. showed a slight lateral rotation motion during the stance phase of gait under loading flexion for 8 subjects [37]. The apparent discrepancy of these results can be attributed to both the different experimental conditions and the choice of coordinate systems. In a study using the same modality and definition of femur and tibia coordinate systems, Zeighami et al. reported an amplitude of medial rotation during the first $20^{\circ}$ of flexion of the knee of $11.8^{\circ}$ in average for ten healthy subjects close to the value obtained in the present study [27].

\subsection{In vitro accuracy}

First, compared to previous studies using the same kind of techniques for other bony structures, the accuracy of the 3D absolute angular and linear position estimation of the tibia was of the same order of magnitude as already reported for the patellofemoral joint [29] or the scapulo-humeral joint [30].

Concerning the femoro-tibial joint, Zeighami et al. reported RMS errors of in average $0.63^{\circ}$ for all rotations and $0.8 \mathrm{~mm}$ for all translations using the same modality (EOS system) and a semi-automatic method of registration [27]. These results are similar to the ones obtained in the present study (Table 2) by using a manual method. In addition, in the present study, the gold standard of the knee kinematics was obtained from clusters screwed in cadaver knees put in motion through a specific device. On the contrary, Zeighami et al. quantified the accuracy with simulated images from 3D models of femur and tibia virtually positioned in different configuration of flexion. We also

Table 3

Standard deviation $\left(S_{R}\right)$ reliability for each of the 6 parameters of the femur and tibia pose in vivo.

\begin{tabular}{|c|c|c|c|c|c|c|c|c|c|c|}
\hline & \multirow{3}{*}{$\begin{array}{l}\text { Knee } \\
\text { positions }\end{array}$} & \multicolumn{9}{|c|}{ In vivo reliability } \\
\hline & & \multicolumn{3}{|c|}{ Femur } & \multicolumn{3}{|c|}{ Tibia } & \multicolumn{3}{|c|}{ Tibia relative to femur } \\
\hline & & $20^{\circ}$ & $40^{\circ}$ & $90^{\circ}$ & $20^{\circ}$ & $40^{\circ}$ & $90^{\circ}$ & $20^{\circ}$ & $40^{\circ}$ & $90^{\circ}$ \\
\hline & Ty (mm) & 0.5 & 0.6 & 0.6 & 0.8 & 0.7 & 0.9 & 1.0 & 1.1 & 1.7 \\
\hline & $\mathrm{Tz}(\mathrm{mm})$ & 0.7 & 0.7 & 0.9 & 0.9 & 1.1 & 1.4 & 1.2 & 1.5 & 1.8 \\
\hline Angular parameters & $\operatorname{Rx}\left({ }^{\circ}\right)$ & 0.4 & 0.4 & 0.7 & 0.4 & 0.9 & 1.4 & 1.0 & 1.4 & 2.1 \\
\hline
\end{tabular}


Table 4

Accuracy of each angular and linear parameters in the current study, compared to results of 2D fluoroscopy studies [4,12,13].

\begin{tabular}{|c|c|c|c|c|c|c|c|c|}
\hline & \multicolumn{3}{|c|}{ Accuracy regarding angular parameters } & \multicolumn{3}{|c|}{ Accuracy regarding linear parameters } & \multirow{2}{*}{$\begin{array}{l}\text { Experimental } \\
\text { conditions }\end{array}$} & \multirow{2}{*}{$\begin{array}{l}\text { Range of } \\
\text { flexion }\end{array}$} \\
\hline & $\mathrm{Rx}\left({ }^{\circ}\right)$ & $\operatorname{Ry}\left({ }^{\circ}\right)$ & $\mathrm{Rz}\left({ }^{\circ}\right)$ & $\mathrm{Tx}(\mathrm{mm})$ & Ty (mm) & $\mathrm{Tz}(\mathrm{mm})$ & & \\
\hline Current study & 0.3 & -0.4 & -0.1 & 1.2 & 1.6 & -0.3 & Quasi static conditions & $0^{\circ}$ to $90^{\circ}$ \\
\hline Acker et al. (2011) & 0.3 & -1.1 & -2.1 & 1.3 & -0.5 & 1.9 & & $0^{\circ}$ to $80^{\circ}$ \\
\hline Mafhouz et al. (2003) & -0.1 & 0 & 0.3 & 0 & -0.1 & 1.1 & Dynamic conditions & $0^{\circ}$ to $120^{\circ}$ \\
\hline Lebel et al. (2011) & 0.1 & 0.5 & 0.1 & -0.5 & 0.1 & 0.7 & & $0^{\circ}$ to $105^{\circ}$ \\
\hline
\end{tabular}

reported the value of accuracy for each flexion pose. It allowed to observe the deterioration of this accuracy when the bones superposed showing the importance of quantifying this accuracy in physiologic conditions. Finally, it can be noticed that the semi-automatic process remained time consuming with a 90 min process for 5 poses against 20 min for 4 poses with our manual method.

In the literature, several studies investigated the accuracy of the in vitro estimation of tibia and femur poses from $2 \mathrm{D}$ fluoroscopy associated with the 3D model against optoelectronic devices $[4,12,13]$ (Table 4). However, the comparison to previous results must be made with care as several conditions differed. First, contrary to the present study based on the analysis of 6 in vitro knee specimens, these studies relied up on a unique cadaver specimen implanted with a knee prosthesis which CAD description was matched with radiographic contours. Secondly, fluoroscopy allows dynamic analysis of the flexion of the instrumented knee contrary to stereoradiography. Only Acker et al. proposed to evaluate the method on 6 static poses. Finally, the reported pose could be the one of the femur [4], of the tibia (present study) or of the knee joint $[12,13]$. The average difference between each position parameter and the cluster-based gold standard is summarized in Table 4. The inaccuracy of the estimation of the out-of-plane translation in fluoroscopic studies was pointed out in all studies. Our results show that the frontal view of the stereoradiography allows us to overcome this limit with an accuracy of the translation Tz clearly enhanced (average on all tibia positions: $-0.3 \mathrm{~mm}$ ).

The accuracy of angular parameters, obtained by our method, is equivalent to the one obtained in fluoroscopic studies [4,12]. In the same time, linear parameters in the sagittal plane (Tx and Ty) are slightly less accurate. These results must be put in perspective with the conditions of the present study which are less favorable than the one of fluoroscopic studies. Indeed, considered specimens are not implanted and the registered 3D model, obtained from reconstruction, is obviously less accurate than the CAD model of the prosthesis. Moreover, our study involves 6 specimens, thus taking into account the interindividual variability.

When analyzing in more details the results obtained for the different knee flexion poses $\left(20^{\circ}\right.$ and $\left.40^{\circ}\right)$ reported in Table 2 , we can observe that the accuracy of the positional parameters in the sagittal plane ( $\mathrm{Rz}, \mathrm{Tx}$ and Ty) are better in the $20^{\circ} \mathrm{knee}$ flexion pose than in the $40^{\circ}$ knee flexion pose. At $40^{\circ}$ of flexion, there is an overlapping of the distal part of the femur with the proximal part of the tibia due to the experimental apparatus in which the femur is fixed and faced the radiographic plane in the frontal view. Moreover, in this position, the distal part of the tibia was not visible on the sagittal view due to the dimensions of the image. It suggests that accuracy of the method could be enhanced by using a mobile femur device which would prevent this limit.

To sum up, the proposed method, allows us to estimate the tibia bone pose with an accuracy equivalent to those reported in studies combining 2D fluoroscopy and 3D modeling of the joint. Currently, in comparison with studies reported in the literature which focused on cadaver TKA knees, the present contribution performed this estimation for non-implanted knees.

The use of a CAD model corresponds to the best case scenario with high contrasted, well defined edges due to the dense metal components. To transfer the method to non-implanted knee, the alternative is to obtain the $3 \mathrm{D}$ model with the CTscan, which increases the overall radiation dose of the protocol. By comparison, based on recent studies, the radiation doses of digital radiography and CT scanner are respectively 20 times [39] and 74\% [40] higher than biplanar radiography with EOS system. Therefore, stereoradiography with low dose radiation system [41] represents a valuable alternative when considering in vivo kinematics evaluation. Indeed, both reconstruction and registration can be derived from the same modality. In addition, the dimension of the images and the presence of an additional view compared to fluoroscopy ensure, as showed by our result, a better accuracy in the out-of-plane parameters estimation.

\subsection{In vivo repeatability}

For in vivo data acquisition, the protocol was slightly different than for in vitro set up as both femur and tibia of healthy knees were mobile. Thus, it has been possible to individualize the repeatability of $3 \mathrm{D}$ tibia registration from $3 \mathrm{D}$ femur registration on bi-planar views.

The repeatability evaluated in vivo was shown to be the best for the flexion/extension of the tibia relative to the femur $(95 \%$ $\mathrm{CI}$ of $\mathrm{Rz}$ inferior to $0.7^{\circ}$ ). From the results, we also showed that the registration of the femur is better than the one of the tibia in particular as concerns the medial/lateral rotation and the translations in the three directions. For the rotation, this can be explained by the shape of the distal extremity of the femur, which facilitates its identification on the frontal view compared to the proximal part of the tibia. Femoral condyles are easier to discriminate than tibial plateaus on this view. These results are in favor of a stereoradiographic protocol in which the tibia would remain fixed throughout the sequence.

In the current study, the maximum uncertainty was found for the rotation around the longitudinal axis ranging from $3.6^{\circ}$ to $5.5^{\circ}$ (CI 95\%) according to the knee flexion pose. The re- 
peatability of the medial/lateral rotation estimation from our registration technique is of the same order as the one of the lateral/medial rotation estimated from the reconstruction of the $3 \mathrm{D}$ model in standing position, reported to be lower than $4^{\circ}$ or $5^{\circ}$ in previous studies $[19,22]$.

As concerns translations, our results showed values lower than $1.8 \mathrm{~mm}\left(S_{R}\right)(\mathrm{CI} 95 \%: 3.6 \mathrm{~mm})$ which is consistent with the results of Zhang et al. when computing the linear position of the humerus relative to the scapula from a similar method (CI 95\% lower than $3.4 \mathrm{~mm}$ ) [30].

The comparison of the results obtained for each knee flexion pose showed that the registration is less reliable when the knee is $90^{\circ}$ flexed. In this pose, radiographic images showed a superposition of the two bones, since the joint space width decreases with flexion [42].

For semi-automatic registration, the repeatability of the method was investigated by Kanhonou et al. and was shown to be very good with values inferior to $1 \mathrm{~mm}$ and $1^{\circ}$ [28]. These values are slightly better than ours which could be expected due to the use of image processing to assist the registration but should be completed by an evaluation of the accuracy in physiologic configurations to ensure the unbiased nature of the measurement.

Compared to methods of quantification of the knee kinematics reported in the literature, the method proposed in the current paper has several advantages. Currently, the gold standard method to assess knee kinematics in vivo is through a bi-planar fluoroscopy [6]. This technic necessitates a 3D model of the studied knee. In the absence of a prosthetic knee, this model is often obtained from CT-scan [3]. In the present study, the reconstruction of the 3D model and the registration can be realized with the same modality. Indeed, the overall method necessitates also a reconstruction step which accuracy was already quantified [22]. However the influence of the morphology was not evaluated in this Assi et al. study. Moreover, contrary to marker based 3D motion analysis, the method is not sensitive to skin movement during the flexion of the knee.

Most often, clinical quantification of knee kinematics is performed by motion capture of skin markers inducing a high uncertainty due to soft tissue artifact $[10,43,44]$. However, it can be expected that knee pathologies such as osteoarthritis or ligament injuries would modify the kinematics of the femoro-tibial joint [27]. The quantification of the kinematic pattern alteration, in particular for translation modification, requires very accurate techniques.

\section{Limits of the study}

This study has some limitations. First, the different knee flexion poses were performed sequentially resulting in different muscles activation compared to a dynamic condition. This could have slightly modified the sequential kinematics of the femoro-tibial joint, in accordance with the results of Marin et al. [45]. However, the consistency of the sequential kinematics with previous results from dynamic analysis allows us to moderate this comment. Second, the number of subjects could be increased to better represent the interindividual variability of knee kinematics. Third, the method is manual and the time required for the whole process is around 20 min (reconstruction and registrations in every position). Finally, the last limitation of the study is that the effective dose was not directly measured by a dosimeter preventing direct comparison with other modalities of imaging. Indeed, the dose was not the main focus of the study but a gross estimation of the emitted dose was made. This yields values inferior to $2 \mathrm{mGy}$ per subject are in accordance with the protocol approved by the ethics committee and consistent with the low dose radiographic system.

\section{Conclusion}

The EOS system offers the possibility to obtain a 3D subjectspecific model of the whole lower limb from bi-planar X-rays captured in weight-bearing conditions while limiting the invasiveness of the procedure. The low dose associated with the $\mathrm{X}$-rays acquisitions using the EOS system allows multiple acquisitions of the joints for a sequence of poses particularly in vivo. The results of the current study confirmed the accuracy and the reliability of the combination of $3 \mathrm{D}$ reconstruction and manual registration of the femur and the tibia for different knee flexion poses to estimate the knee sequential kinematics. By comparing with other methods of motion capture, we showed the feasibility of using the current EOS-based method for clinical examination of the femoro-tibial joint.

\section{Conflict of interest statement}

There is no conflict of interest to report.

\section{Acknowledgements}

Thanks to Guillaume Dubois for his technical support. Authors thank the ParisTech BiomecAM chair program on subjectspecific musculoskeletal modeling.

\section{References}

[1] Andriacchi TP, Mündermann A, Smith RL, Alexander EJ, Dyrby CO, Koo S. A framework for the in vivo pathomechanics of osteoarthritis at the knee. Ann Biomed Eng 2004;32(3):447-57.

[2] Farrokhi S, Tashman S, Gil AB, Klatt BA, Fitzgerald GK. Are the kinematics of the knee joint altered during the loading response phase of gait in individuals with concurrent knee osteoarthritis and complaints of joint instability? A dynamic stereo X-ray study. Clin Biomech (Bristol, Avon) 2012;27(4):384-9.

[3] You BM, Siy P, Anderst W, Tashman S. In vivo measurement of 3-D skeletal kinematics from sequences of biplane radiographs: application to knee kinematics. IEEE Trans Med Imaging 2001;20(6):514-25.

[4] Mahfouz MR, Hoff WA, Komistek RD, Dennis DA. A robust method for registration of three-dimensional knee implant models to two-dimensional fluoroscopy images. IEEE Trans Med Imaging 2003;22(12):1561-74.

[5] Li G, Wuerz TH, DeFrate LE. Feasibility of using orthogonal fluoroscopic images to measure in vivo joint kinematics. J Biomech Eng 2004; 126(2):314-8.

[6] Li G, Van de Velde SK, Bingham JT. Validation of a non-invasive fluoroscopic imaging technique for the measurement of dynamic knee joint motion. J Biomech 2008;41(7):1616-22. 
[7] Miranda DL, Rainbow MJ, Crisco JJ, Fleming BC. Kinematic differences between optical motion capture and biplanar videoradiography during a jump-cut maneuver. J Biomech 2013;46(3):567-73.

[8] Lafortune MA. Three-dimensional kinematics of the human knee during walking. J Biomech 1992;25(4):347-57.

[9] Chiari L, Della Croce U, Leardini A, Cappozzo A. Human movement analysis using stereophotogrammetry. Part 2: instrumental errors. Gait Posture 2005;21(2):197-211.

[10] Stagni R, Fantozzi S, Cappello A, Leardini A. Quantification of soft tissue artefact in motion analysis by combining 3D fluoroscopy and stereophotogrammetry: a study on two subjects. Clin Biomech 2005;20(3):320-9.

[11] Garling EH, Kaptein BL, Mertens B, Barendregt W, Veeger HEJ, Nelissen RGHH, et al. Soft-tissue artefact assessment during step-up using fluoroscopy and skin-mounted markers. J Biomech 2007;40(Suppl 1):S18-24. https://doi.org/10.1016/j.jbiomech.2007.03.003. J Biomech 2008;41(10):2332-3.

[12] Acker S, Li R, Murray H, St John P, Banks S, Mu S, et al. Accuracy of single-plane fluoroscopy in determining relative position and orientation of total knee replacement components. J Biomech 2011;44(4):784-7.

[13] Lebel BP, Pineau V, Gouzy SL, Geais L, Parienti J-JM, Dutheil J-JP, et al. Total knee arthroplasty three-dimensional kinematic estimation prevision. From a two-dimensional fluoroscopy acquired dynamic model. Orthop Traumatol, Surg Res 2011;97(2):111-20.

[14] Johal P, Williams A, Wragg P, Hunt D, Gedroyc W. Tibio-femoral movement in the living knee. A study of weight bearing and non-weight bearing knee kinematics using interventional. J Biomech 2005;38:269-76.

[15] Von Eisenhart-rothe R, Siebert M, Bringmann C, Vogl T. A new in vivo technique for determination of 3D kinematics and contact areas of the patello-femoral and tibio-femoral joint. J Biomech 2004;37:927-34.

[16] Hill PF, Vedi V, Williams A, Iwaki H, Pinskerova V, Freeman MAR. Tibiofemoral movement 2: the loaded and unloaded living knee studied by MRI. 2000 .

[17] Al Hares G, Eschweiler J, Rademacher K. Combined magnetic resonance imaging approach for the assessment of in vivo knee joint kinematics under full weight-bearing conditions. Proc Inst Mech Eng $\mathrm{H}$ 2015;229(6):439-51.

[18] Shapiro LM, Gold GE. MRI of weight bearing and movement. Osteoarthr Cartil 2012;20(2):69-78.

[19] Chaibi Y, Cresson T, Aubert B, Hausselle J, Neyret P, Hauger O, et al. Fast 3D reconstruction of the lower limb using a parametric model and statistical inferences and clinical measurements calculation from biplanar X-rays. Comput Methods Biomech Biomed Engin 2012;15(5):457-66.

[20] Dubousset J, et al. Une nouvelle imagerie ostéo-articulaire basse dose en position debout : le système EOS. Radioprotection 2005;40(2):245-55.

[21] Folinais D, Thelen P, Delin C, Radier C, Catonne Y, Lazennec JY. Measuring femoral and rotational alignment: EOS system versus computed tomography. Orthop Traumatol, Surg Res 2013;99(5):509-16.

[22] Assi A, Chaibi Y, Presedo A, Dubousset J, Ghanem I, Skalli W. Threedimensional reconstructions for asymptomatic and cerebralpalsy children's lower limbs using a biplanar X-ray system: a feasibility study. Eur J Radiol 2013;82(12):2359-64.

[23] Than P, Szuper K, Somoskeöy S, Warta V, Illés T. Geometrical values of the normal and arthritic hip and knee detected with the EOS imaging system. Int Orthop 2012;36(6):1291-7.

[24] Bendaya S, et al. Healthy vs. osteoarthritic hips: a comparison of hip, pelvis and femoral parameters and relationships using the $\operatorname{EOS}^{\circledR}$ system. Clin Biomech (Bristol, Avon) 2015;30(2):195-204.

[25] Südhoff I, Van Driessche S, Laporte S, de Guise Ja, Skalli W. Comparing three attachment systems used to determine knee kinematics during gait. Gait Posture 2007;25(4):533-43.

[26] Jerbi T, Burdin V, Roux C, Stindel E. 2D-3D registration in the frequency domain for the study of the movement in quasi-static condition: pilot study on the knee joint. IRBM 2012;33:18-23.
[27] Zeighami A, Kanhonou M, Hagemeister N, Lavoie F, de Guise JA. Tibiofemoral joint contact in healthy and osteoarthritic knees during quasi-static squat: a bi-planar X-ray analysis. J Biomech 2017;53:178-84.

[28] Kanhonou M, Cresson T, Lavoie F, Clement J, Hagemeister N, de Guise JA, et al. A method to study 3D knee pseudo-kinematics using lowdose stereo-radiography during static squat. Comput Methods Biomech Biomed Eng 2014;17(S1):138-9.

[29] Dagneaux L, Thoreux P, Eustache B, Canovas F, Skalli W. Sequential 3D analysis of patellofemoral kinematics from biplanar $\mathrm{X}$-rays: in vitro validation protocol. Orthop Traumatol, Surg Res 2015;101(7):811-8.

[30] Zhang C, et al. Investigation of 3D glenohumeral displacements from 3D reconstruction using biplane X-ray images: accuracy and reproducibility of the technique and preliminary analysis in rotator cuff tear patients. J Electromyogr Kinesiol 2016;29:12-20.

[31] Azmy C, Guérard S, Bonnet X, Gabrielli F, Skalli W. EOS orthopaedic imaging system to study patellofemoral kinematics: assessment of uncertainty. Orthop Traumatol, Surg Res 2010;96(1):28-36.

[32] Rochcongar G, et al. A new method for the evaluation of the end-to-end distance of the knee ligaments and popliteal complex during passive knee flexion. Knee 2016;23(3):420-5.

[33] Quijano S, Serrurier A, Aubert B, Laporte S, Thoreux P, Skalli W. Threedimensional reconstruction of the lower limb from biplanar calibrated radiographs. Med Eng Phys Dec. 2013;35(12):1703-12.

[34] Schlatterer B, Suedhoff I, Bonnet X, Catonne Y, Maestro M, Skalli W. Skeletal landmarks for TKR implantations: evaluation of their accuracy using EOS imaging acquisition system. Orthop Traumatol, Surg Res 2009;95(1):2-11.

[35] Pillet H, Sangeux M, Hausselle J, El Rachkidi R. A reference method for the evaluation of femoral head joint center location technique based on external markers. Gait Posture 2014;39(1):655-8.

[36] Akbarshahi M, Schache AG, Fernandez JW, Baker R, Banks S, Pandy MG. Non-invasive assessment of soft-tissue artifact and its effect on knee joint kinematics during functional activity. J Biomech 2010;43(7):1292-301.

[37] Benoit DL, Ramsey DK, Lamontagne M, Xu L. Effect of skin movement artifact on knee kinematics during gait and cutting motions measured in vivo. Gait Posture 2006;24:152-64.

[38] Tsai T, Lu T, Kuo M, Lin C. Effects of soft tissue artifacts on the calculated kinematics and kinetics of the knee during stair-ascent. J Biomech 2011;44(6):1182-8.

[39] Abrisham SMJ, Bouzarjomehri F, Nafisi-Moghadam R, Sobhan MR, Gadimi M, Omidvar F. A comparison of patients absorption doses with bone deformity due to the EOS imaging and digital radiology. Arch Bone Jt Surg 2017;5(3):145-8.

[40] Ben Abdennebi A, Aubry S, Ounalli L, Fayache MS, Delabrousse E, Petegnief Y. Comparative dose levels between CT-scanner and slotscanning device (EOS system) in pregnant women pelvimetry. Phys Med 2017;33:77-86.

[41] Melhem E, Assi A, El Rachkidi R, Ghanem I. EOS ${ }^{\circledR}$ biplanar X-ray imaging: concept, developments, benefits, and limitations. J Child Orthopaedics 2016;10(1):1-14.

[42] Reichmann WM, Maillefert JF, Hunter DJ, Katz JN, Conaghan PG, Losina E. Responsiveness to change and reliability of measurement of radiographic joint space width in osteoarthritis of the knee: a systematic review. Osteoarthr Cartil 2011;19(5):550-6.

[43] Leardini A, Chiari A, Della Croce U, Cappozzo A. Human movement analysis using stereophotogrammetry. Part 3. Soft tissue artifact assessment and compensation. Gait Posture 2005;21(2):212-25.

[44] Sangeux M, Marin F, Charleux F, Dürselen L, Ho Ba Tho MC. Quantification of the 3D relative movement of external marker sets vs. bones based on magnetic resonance imaging. Clin Biomech 2006;21(9):984-91.

[45] Marin F, Sangeux M, Charleux F, Ho Ba Tho MC, Dürselen L. Can a finite set of knee extension in supine position be used for a knee functional examination? J Biomech 2006;39(2):359-63. 